\title{
Criterios para evaluar la calidad de las fuentes de información en Internet
}

\author{
José A. Salvador Oliván \\ Departamento de Ciencias de la Documentación \\ e Historia de la Ciencia \\ Universidad de Zaragoza \\ E-mail: jaso@posta.unizar.es \\ José Ma Angós Ullate \\ Centro de Documentación Científica \\ Universidad de Zaragoza \\ E-mail:angos@posta.unizar.es \\ María Jesús Fernández Ruiz \\ Centro de Documentación \\ Ayuntamiento de Zaragoza \\ E-mail:mjferuiz@posta.unizar.es
}

\subsection{Resumen}

La disponiblidad creciente de Internet permite buscar y encontrar información de cualquier parte del mundo. Además, el desarrollo de la World Wide Web ha hecho que dicha red sea más fácil de usar, tanto para buscar información como para publicarla en formato electrónico. Debido a la gran cantidad de información disponible y a sus diferentes niveles de calidad y fiabilidad, se hace necesario evaluar la información recuperada. El objetivo de este trabajo es proporcionar una lista de los items e indicadores más utilizados por diversos autores para evaluar la calidad de la información y de los sitios Web. (Autor)

Palabras Clave: Recursos de la World Wide Web. Evaluación. Calidad de la información.

\subsection{Abstract}

The availability and growth of the Internet offers the opportunity to find information from all over the world. In addition, the development of the World Wide Web has made the Internet easier to use, both for finding information and for publishing it electronically. Because so much information is available and because that information can result in a wide range of quality, it is necessary to 
evaluate what you find. This study discusses and shows the indicators of information and web site quality and the criteria more frequently used to assess information found on the Internet. (Author)

Keywords: World Wide Web Resources. Evaluation. Information quality.

\section{Introducción}

La red Internet se ha convertido en un fenómeno social que ha revolucionado nuestra forma de vida y de trabajar, constituyendo para los usuarios un punto de encuentro y de interrelación personal en cualquiera de sus dimensiones: científica, comercial, lúdica, etc. Esta visión «abierta» de la red y su uso generalizado, ha puesto de manifiesto sus fortalezas y debilidades y provocado diferentes opiniones en cuanto a sus ventajas e inconvenientes.

Pero hay un hecho incuestionable y que a lo largo de su evolución todos hemos podido comprobar: el crecimiento continuo y a un ritmo frenético de los recursos de información que podemos encontrar en Internet, y más concretamente, en la World Wide Web. Cada día que pasa, aparece nueva información, y ello es debido a que la red constituye un excelente medio de difusión y a la vez es muy fácil publicar en él: cualquier persona, organismo o empresa que disponga de un ordenador y de un módem para conectarse a la red, puede poner información (montar una página web) y de hecho, lo que está haciendo es publicar en la red.

Pero a lo anterior se une un factor añadido, y es que se eliminan todas las barreras tradicionales que existían y existen en los medios de edición impresa: a diferencia de éstos, donde para poder publicar hay que pasar por un proceso de evaluación, de filtrado y de revisión, y cumplir con las normas de publicación propias de cada revista o editorial, en la red no existe ningún proceso de screening, no hay nadie que intervenga en la selección y evaluación de los recursos de información, salvo en aquellos casos que corresponden a bases de datos ofrecidas de forma gratuita por sus productores (Eric, Medline, etc.) y los recursos relativos a revistas electrónicas que ofrecen el texto completo de todos o parte de sus artículos publicados (JAMA, Database).

Estos dos factores comentados anteriormente, la facilidad para publicar y la falta de control, ha ocasionado que la World Wide Web se haya convertido en uno de los almacenes más importantes de información del mundo y, no cabe ninguna duda, que en el más utilizado sobre todo por su carácter «gratuito». Así, es evidente que cada vez hay más publicaciones en la Web, pero también que los motores de búsqueda utilizados para buscar información recuperan más basura que otra cosa, debido sobre todo a la gran diversidad de información existente. La información que podemos encontrar en Internet puede ser de muchos tipos (factual, estadísiticas, opiniones, interpretaciones, etc.) y creada para mútliples pro- 
pósitos (persuadir, informar, vender, convencer, presentar un punto de vista, influir en la forma de pensar, etc.), pudiendo existir para cada uno de estos tipos y propósitos en diferentes grados de calidad y fiabilidad. Siempre se ha cuestionado la falta de calidad de la información, debido a la propia naturaleza intrínseca de la red: dinámica, caótica, a menudo desorganizada y con información de dudosa o desconocida calidad, y a que no hay nadie que controle ni gobierne el flujo de la información ni que verifique su calidad.

Esta situación contrasta con la importancia que en el ámbito de las Ciencias de la Documentación y de la Información ha tenido siempre la evaluación tanto de la calidad de la información como de los sistemas recuperación de información, con el objetivo de proporcionar al usuario la información que necesita y que además sea una información útil y de calidad. Por consiguiente, parece claro que cuando buscamos información en la Web, debemos llevar a cabo algún tipo de evaluación para comprobar que la información recuperada tenga un cierto grado de calidad, y en este sentido, el profesional de la documentación se enfrenta a unos nuevos retos como son localizar información de calidad y útil en la red, y evaluar dicha información para verificar su calidad.

En la actualidad, hay dos alternativas para poder conocer algo más sobre la calidad de la información recuperada.

La primera es a través de los diferentes servicios y sitios de revisión incluídos en los propios directorios y motores de búsqueda, como The Argus Clearinghouse, Magellan, Lycos, etc., que evalúan los recursos de información, proporcionando comentarios, ordenando los resultados de la búsqueda según la relevancia, pero suelen aplicar diferentes criterios y escalas de puntuación. Así, The Argus Clearinghouse considera criterios como autoridad, precisión y actualización; otros, tienen en cuenta la facilidad de uso, la organización, su cobertura y atractivo.

Aunque estas herramientas de revisión proporcionan información interesante sobre los recursos de información, y teniendo presente que los diferentes tipos de información que se pueden encontrar en la red pueden satisfacer diferentes necesidades de información, cada vez se considera más relevante realizar un control de calidad aplicado al mundo electrónico de Internet. Por consiguiente, los usuarios querrán y necesitarán revisiones evaluativas, analíticas y dignas de confianza que les ayuden a elegir dentro del gran rango de recursos existentes. Páginas web con información precisa y fiable conviven con páginas de información inexacta, de poca fiabilidad o falsa; además, las diferencias entre estos dos tipos de información puede no ser muy evidente sobre todo para el usuario que no es un experto en ese área temática. Siendo además que la responsabilidad de Internet no recae en ninguna organización o institución, es poco probable que se

Scire. 5 : 2 (jul.-dic. 1999) 99-113. 
establezca un control de calidad universal en Internet, al menos en un futuro cercano, y los usuarios deben prepararse para analizar de forma crítica las fuentes de información que encuentren.

Por ello, la segunda alternativa es que sea el propio usuario o el documentalista quien evalúe la fuente de información de forma más profunda y crítica, analizando si realmente satisface sus necesidades de información, mediante el aprendizaje y aplicación de un conjunto de criterios que ayuden a determinar y evaluar la calidad tanto de los sitios web como de la información que contienen, a discernir lo bueno de lo malo, lo útil de lo inútil, ya que saber cómo juzgar la calidad de los recursos de la red es incluso más importante que saber cómo buscarlos (Solock, 1990).

\section{Objetivo}

El objetivo de este trabajo se centra en este segundo apartado, y no es otro que revisar y analizar aquellos criterios e indicadores más utilizados por diferentes autores en la evaluación de los recursos de información en la Web, dejando claro que no habrá que aplicarlos de forma sistemática a todos los sitios web recuperados cuando se hace una búsqueda con cualquiera de los buscadores, sino que se utilizarán para valorar solamente aquellos recursos que se ajusten a nuestras necesidades de información, para evaluar y comparar criterios como la calidad, fiabilidad, facilidad de uso, etc., y que aquellos recursos que se supone han pasado ya un proceso de revisión (revistas electrónicas, bases de datos online, artículos ya publicados en revistas impresas, etc.) no es necesario evaluarlos.

El uso de estos criterios nos ayudará a separar información de alta calidad de información de baja calidad. Algunos de estos indicadores son exclusivos de la evaluación de recursos en la red, pero otros son los que se utilizan también para la evaluación de recursos impresos. No se trata de hacer una guía exhaustiva de todos los ítems que hay que medir para llevar a cabo una evaluación, sino que lo que se pretende es ver qué aspectos, de forma genérica, hay que tener en cuenta y medir a la hora de evaluar los recursos de información.

Antes de empezar a evaluar una fuente de información, hay que tener presente una serie de cuestiones. En primer lugar, hay que preguntarse qué estamos buscando, qué tipo de información necesitamos, cuál es el objetivo de nuestra búsqueda. Al igual que cuando realizamos una búsqueda de información en una base de datos, y evaluamos los resultados en función de nuestras necesidades de información, también deberemos aplicar este criterio al evaluar las fuentes de información encontradas en Internet, sobre todo teniendo en cuenta la gran variedad de material que podemos encontrar (opiniones, estadísticas, argumentos, hechos, informes, etc.), y esta falta de uniformidad nos lleva a una segunda cues- 
tión a tener en cuenta, y que no es otra que muchas veces no podremos aplicar todos los indicadores o items que se especifican en este trabajo para llevar a cabo el proceso de evaluación. El hecho de tener claro el objetivo y el tipo de información que necesitamos, nos ayudará a discriminar rápidamente información no útil, aunque pueda ser de calidad.

\section{Material y Método}

Se ha realizado una búsqueda de información en los buscadores Altavista, Yahoo y Excite sobre el tema «Evaluación de recursos o fuentes de información en Internet» $\mathrm{y}$ «Calidad de la información en la Web», seleccionando los lugares más relevantes que son los primeros que aparecen en la lista de resultados. Asimismo, se han analizado también aquellos enlaces de esas publicaciones que hacían referencia a la evaluación de la calidad de la información y las compilaciones de bibliografías sobre evaluación de sitios web (Kotlas, 1997) y de fuentes de investigación en Internet (Auer, 1998).

El número total de trabajos publicados en la Web que se han revisado ha sido de 32 (Véase el apartado de Bibliografía). Los indicadores o items utilizados en estos trabajos los hemos agrupado en dos grandes categorías:

- aquellos relacionados con la calidad de la información (contenido), y

- los relacionados con la calidad del sitio (facilidad de acceso y uso o navegación, diseño, etc.).

Esta diferenciación se debe a que no todos los trabajos evalúan estos dos grandes apartados, sino que algunos están orientados a evaluar la calidad de la información, y otros a evaluar la presentación del recurso web.

De estos dos grandes grupos, el que mayor dificultad presenta a la hora de evaluar y medir es el relacionado con la calidad de la información, ya que para medir algo primero hay que definirlo, y definir lo que se considera información de calidad es un tema bastante resbaladizo, ya que implica unos criterios subjetivos y lo que para uno puede ser de utilidad para otro puede no serlo, y por tanto, va a depender de quién es el usuario que juzga y para qué quiere la información.

Lo que se ha hecho en este caso es adaptar algunos criterios de evaluación que caracterizan una información de calidad en los recursos tradicionales: cobertura, actualidad, autoridad, precisión, objetividad, etc., y utilizarlos para evaluar la calidad de la información en Internet; al fin y al cabo, no deja de ser información aunque publicada en un medio no impreso de forma tradicional. Ahora bien, hay que tener presente que no hay un indicador perfecto que nos mida la calidad o el valor de la información. Robert Harris (1997) dice que determinar la calidad de la información es un arte, ya que hay que inferir a partir de un conjunto de indicadores, basados en la utilidad o propósito con el que se quiera utilizar la 
fuente de información. Pocas fuentes de información van a satisfacer todos los criterios, pero el aplicarlos nos será de utilidad para distinguir la información de alta calidad de la de baja calidad, y a mayor calidad, mayor será su valor, si bien el valor de la información está siempre relacionado con la persona y el contexto en el que la información tiene que ser útil.

También, comentar que algunos indicadores son más fáciles de medir y, además, de manera muy rápida; sin embargo, para ver el cumplimiento de otros, se requiere una evaluación mucho más profunda, teniendo en cuenta que algunos están muy relacionados entre sí.

\section{Resultados}

El aspecto relativo a la calidad de la información, la hemos dividido en dos facetas: por una parte, los indicadores relacionados con el autor o responsable del recurso, y por otra, los relacionados con el propio contenido.

\subsection{Autor}

Muy a menudo se relaciona la calidad de información de un documento con el autor o autores del mismo. En el Gráfico I se puede observar los diferentes criterios relacionados con la autoría de la fuente de información, destacando sobre

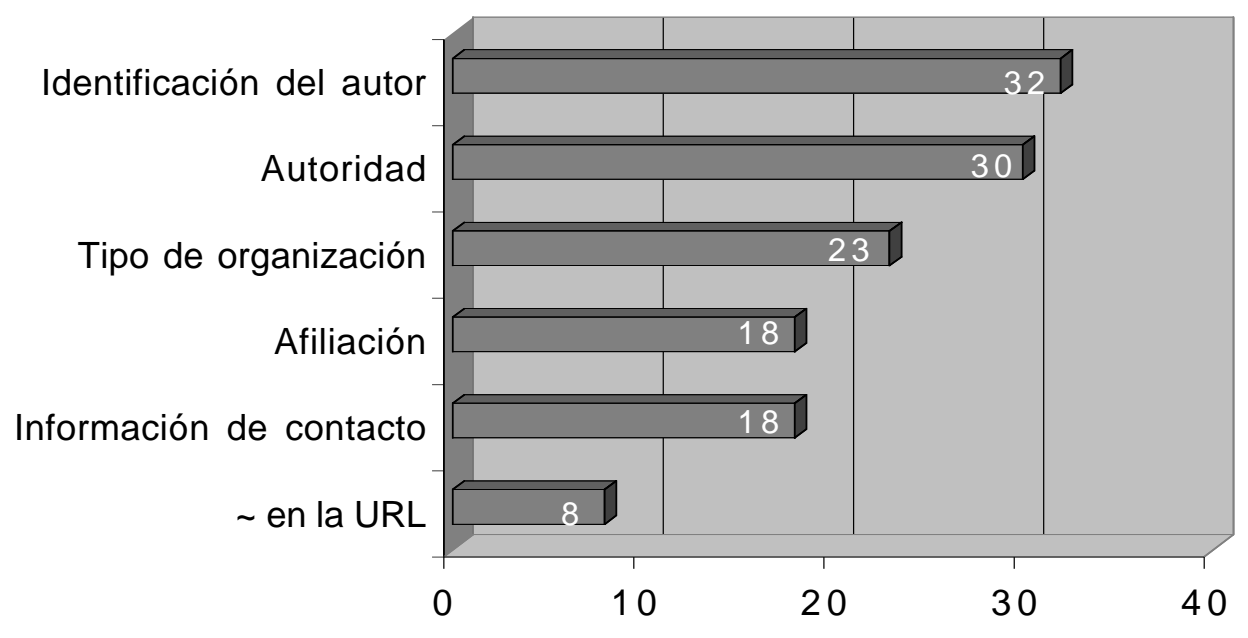

Gráfico I. Criterios de calidad de la información relacionados con el Autor 
todo la Identificación del autor como principal item que no debe de faltar nunca en un recurso de información; también se le da bastante importancia para evaluar la calidad de la información a datos sobre la autoridad de la persona o de la organización que la ha creado.

- Identificación del autor: como se puede ver en el Gráfico I, hay una total coincidencia en que el autor, bien sea un individuo u organización, debe estar plenamente identificado. Generalmente, el autor o el organismo responsable del recurso aparece en la cabecera o en el pie de la página; si no aparece en ningún sitio, se puede determinar analizando hacia atrás la URL. Si la página no viene firmada y no se puede determinar qué institución o personas son los autores o promotores, esa información es tan anónima como una página arrancada de un libro (Kirk, 1996). Una información en la que no esté identificado el autor, evidentemente indica que la fuente no es fiable y una falta de credibilidad. No se puede evaluar algo que no se puede verificar, y por consiguiente, no es aconsejable utilizar información de esta naturaleza.

- Relacionada con la identificación del autor, también se le da mucha importancia a la autoridad que tiene sobre el tema: título, posición, reputación, experiencia y curriculum. La calificación o autoridad del autor es un indicador potente de la fiabilidad y credibilidad de la información. Se pueden encontrar estos datos biográficos en la página web; en el caso de que no estén presentes, podemos utilizar diferentes herramientas como Who's Who, Biography Index, o enciclopedias biográficas y directorios para determinar quién es el autor y sus credenciales. También se puede buscar el nombre del autor en algún buscador como Altavista, lo que nos puede llevar a otra información o a otras páginas del mismo autor.

- Cuando el autor o el espónsor sea un organismo, qué tipo de organización es: educativa, comercial, gubernamental. Examinar el dominio: .edu, .com. .gov, .org, .net. El motivo es que muchas veces no se indica el patrocinador o promotor, y el tipo de información y el propósito es diferente según el tipo de organización. ¿Es seria y de confianza la organización? ¿Qué reputación tiene?

- En poco más de la mitad de los trabajos, se concede importancia a la afiliación del autor, y a que aparezca información de contacto con el autor: email, teléfono, fax y dirección, para poder solicitar más información sobre su trabajo o sobre su curriculum profesional. Si no se conoce la afiliación del autor, se puede intentar conocer retrocediendo en la URL hasta llegar a la página del servidor. 
- En 8 ocasiones, se hace mención a que cuando aparece el carácter $\sim$ en la URL, significa una página personal, y por consiguiente, este tipo de recursos deberían verse con precaución, ya que se considera que las fuentes de información de universidades, organizaciones conocidas, centros de investigación, etc., son más fiables. Si bien, existen autores que también dicen que no hay que considerar la fuente de información únicamente por las características de su URL.

\subsection{Características de la información}

En el Gráfico II se muestran los criterios más frecuentes utilizados para valorar la calidad de la información, destacando sobre todo que la información debe ser actual y en 29 trabajos hacen referencia que el objetivo y audiencia a quién va dirigida debe de figurar en el recurso. A continuación, se comentan todos los items relacionados con la calidad del contenido:

- Inclusión de la fecha de creación y de revisión o de última actualización, que indica la actualidad y/o actualización de la información. Todos los trabajos hacen referencia a este item, importante sobre todo en recursos de información sobre temas que pueden cambiar de forma rápida, especialmente en medicina, ciencia, tecnología, etc. ¿Es actualizado con frecuencia el recurso o, por el contrario, es un recurso estático?. Si se necesita infor-

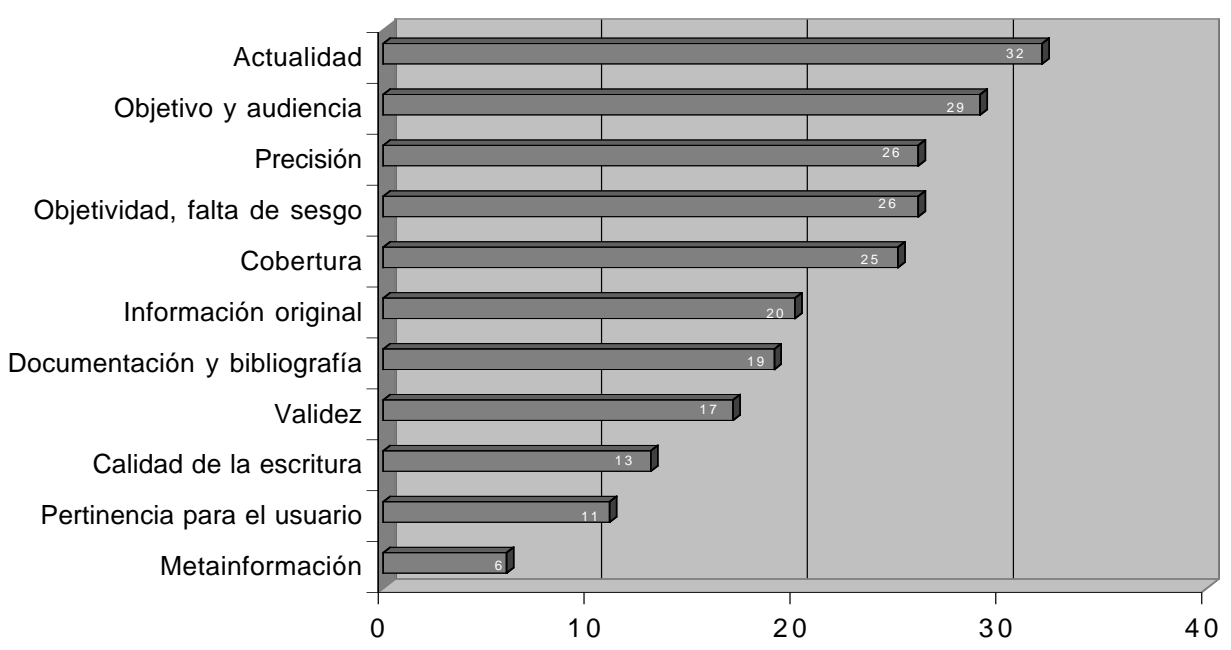

Gráfico II. Criterios de calidad de la información relacionados con las características de la información. 
mación actual, revisar las fechas de creación y de revisión mostradas en las páginas web para determinar su actualidad. En el caso de que no se de ninguna fecha, se puede ver el directorio en el que reside el documento y leer la fecha de la última modificación, a través de Información del documento con el navegador.

- Objetivo y audiencia. ¿A quién va dirigida? ¿Al público general o a un público especializado. Es la fuente demasiado elemental, demasiado técnica, demasiado avanzada o es la adecuada a las necesidades del usuario. ¿Con qué propósito se ha creado la información? Persuadir, informar, explicar, divertir. Se deben declarar las intenciones en la página inicial, o proporcionar un enlace desde la página inicial.

- Precisión y exactitud: Aunque en 26 trabajos se hace referencia a este item, para valorarlo hay que tener en cuenta otras características como la objetividad, validez, siendo el cumplimiento de éstas las que resultan en una información precisa y exacta. La valoración de este ítem requiere un proceso de evaluación y de revisión similar al que sufren los recursos impresos por parte de los editores y evaluadores, y a veces será necesario examinar otras fuentes de información, bien sea en la red o de forma impresa.

- Objetividad, falta de sesgo, equilibrio: Relacionada con el propósito con el que ha sido escrita la información, hay que tenerla en cuenta a la hora de interpretar y usar la información. Internet se ha convertido en una herramienta muy importante de publicidad y de márketing, por lo que es aconsejable preguntarse por qué el autor ha puesto esa información en la red. ¿Cómo es el tono del lenguaje utilizado?, ¿es equilibrado o despierta emociones?. Datos sobre la afiliación o autoría del recurso nos alertarán sobre un posible sesgo o un punto de vista parcial de la información dada.

- Cobertura: hay que determinar el nivel de profundidad y de exhaustividad con que se cubre el tema en cuestión, y si la información es completa, qué aspectos se cubren, con qué nivel de detalle. El que un sitio se compare con otros sobre el mismo tema, nos puede ayudar a determinar el grado con que la información presentada cubre el tema abordado, y aportar diferentes puntos de vista.

- Hay que saber distinguir si es información primaria o secundaria, si es un sitio con información original, que contribuye a algo único sobre el tema, o apenas contiene información y es un puntero que contiene sólo enlaces. Debido a la gran circularidad de enlaces, existen páginas que sólo contienen enlaces y no añaden nada nuevo. Los dos tipos de recursos de información pueden ser útiles, pero hay que saber distinguirlos. En el caso de que la información no sea original (total o en parte), 
- Qué documentación se aporta y apoya la información dada, bien en forma de blibiografía y/o en forma de enlaces a otras páginas. Especialmente importante cuando la información se base en datos numéricos o estadísticas. También importante cuando se trate de opiniones o conclusiones para poder dirigirse a las fuentes que las avalan o apoyan, ya que el citar éstas aumenta la credibilidad de la información.

- Validez: Hace referencia al grado de confianza que nos merece la información, y si se puede confirmar con hechos aceptados. ¿Se indican las fuentes de datos y la metodología utilizada?. Este aspecto es muy importante sobre todo en información relacionada con la investigación, donde se aplica el método científico. Hay que conocer cómo se han obtenido los datos, de dónde, qué métodos se han utilizado, y si son adecuados.

- Calidad de la escritura y sintaxis: buena gramática, sin faltas de ortografía. El hecho de que un texto esté bien escrito es importante para que el proceso de la comunicación se realice de forma clara. Los errores de sintaxis y de gramática pueden indicar una falta de cuidado en la redacción del texto.

- Relevancia y pertinencia para las necesidades de información del usuario. ¿Satisface sus necesidades de información? Este es un criterio totalmente subjetivo que debe responder únicamente el usuario.

- Por último, y sólo en seis ocasiones, se concede importancia a la metainformación (información sobre la información). Esta constituye un medio rápido de saber si la información es de calidad y de utilidad. Hay dos tipos: metainformación de resumen, que incluye resúmenes, sumarios o tablas de contenido y que nos permiten conocer el contenido sin tener que leer el documento completo; y metainformación evaluativa, que a través de puntuaciones, revisiones o comentarios proporciona un análisis del contenido. Conforme aumenta el número de recursos de información, cada vez será más importante poder acceder a metainformación de alta calidad. ¿Existen revisiones del sitio?.

\subsection{Calidad del Sitio Web}

En este apartado, los aspectos a considerar son ya relevantes y específicos de la información puesta en la red y no de la información impresa tradicional. En el Gráfico III se observa que hay una total coincidencia en que el mantenimiento y actualización del sitio es el indicador más frecuentemente utilizado para valorar la calidad del propio sitio Web. Sigue en orden de importancia el propósito con el que se ha creado, y menos frecuentemente, aspectos como la operatividad y relevancia de los enlaces y aquellos relacionados con el diseño y estructura del recurso de información. 
- Mantenimiento y actualización del sitio, especificando la fecha de creación y cuándo se ha revisado por última vez. ¿Se mantiene el recurso de forma regular, es dinámico?, o por el contrario, ¿es estático?. Para que un recurso sea operativo debe mantenerse y actualizarse periódicamente. Aquellos sitios no actualizados, suelen contener enlaces no operativos o sustituídos pos otras páginas más actuales.

- Audiencia, a quién va dirigido el sitio, la presencia de «About this site», cuál es su propósito. ¿Está indicado claramente a qué público o usuarios va dirigido el sitio?. La información tiene que poder ser entendida y asimilada por el usuario al que va dirigido el sitio. Información demasiada compleja o demasiado simple, generalmente no es útil. Los sitios de confianza deberían declarar sus intenciones en la página inicial, o proporcionar un enlace expreso sobre ellas.

- Si existen enlaces a otros sitios, ¿cómo trabajan?, ¿están actualizados? ¿son relevantes, selectivos y apropiados para el tema a que hacen referencia? La calidad de los enlaces hace referencia a la utilidad de éstos ( ¿son sólo listas de listas o son punteros a información más fundamental?), y a si están anotados o marcados de forma clara, visible y despejada.

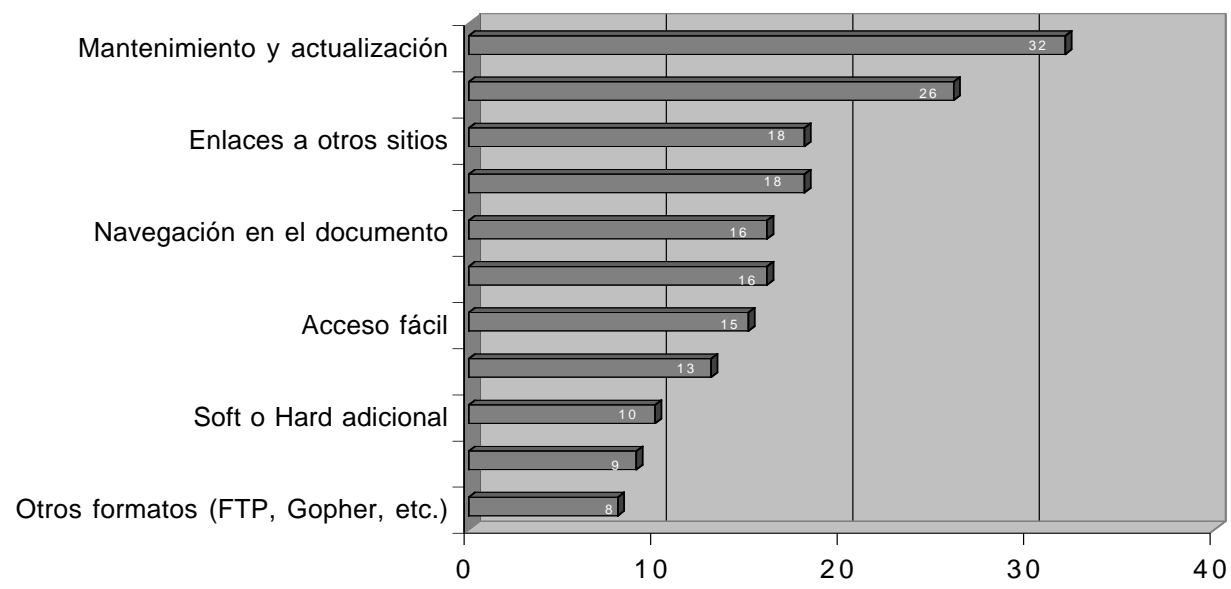

Gráfico III. Criterios relacionados con la calidad del sitio Web

Scire. 5 : 2 (jul.-dic. 1999) 99-113. 
- Diseño y estructura de las páginas web: niveles, estructuras. Se refiere a cómo está organizada y estructurada la información. El diseño de la página es crucial y deberá ser claro y lógico, permitiendo un acceso fácil no sólo al contenido del sitio sino a todas sus partes: propósito, autoridad, cobertura, actualización, etc. Un buen diseño de las páginas Web ayudará a utilizarlas y a establecer la diferencia entre recomendar un recurso o rechazarlo.

- Navegación dentro del documento. Facilidad para desplazarse y localizar información, con capacidad de búsqueda dentro del documento (amigabilidad). ¿Tienen enlaces que envían al principio y al final, o a la página anterior y posterior? ¿Hay un mapa del sitio o una tabla del contenido?. ¿Es intuitivo el sitio? Si el sitio no es fácil de utilizar, probablemente los usuarios no lo utilicen más de una vez. Es muy importante que los documentos se puedan explorar con facilidad, y esto está relacionado con la estructura y organización: diseño de menús, índices, tablas de contenido, funciones de búsqueda y ayuda online. Si un sitio no es fácil de navegar y de usar, es poco probable que lo usuarios lo utilicen.

- Estabilidad: ¿Es un sitio estable a lo largo del tiempo? Si la URL cambia, los editores fiables suelen establecer marcadores de la nueva localización de las páginas web.

- Acceso al sitio y facilidad de uso: Antes de poder entrar a valorar la calidad de un recurso, es necesario localizar y entrar en el servidor que contiene la información. Por consiguiente, se puede decir que es la primera impresión que recibe el usuario cuando entra al sitio: facilidad de conexión, identificación del sitio, descarga rápida de la página, limitaciones de acceso, etc.

- Las ilustraciones y gráficos que aparecen ¿añaden valor al sitio? ¿o son sólo decorativas?. Si se utiliza audio y vídeo, ¿son apropiados para el objetivo del sitio?

- ¿Se requiere software o hardware adicional, o las últimas versiones de los navegadores?; ¿hay que descargar software para utilizarlo?. Aunque el sitio sea de calidad, puede que no sea accesible para muchos usuarios que no tengan el software adecuado para ver el recurso. Algunos autores hacen hincapié en que los sitios de calidad deberían poder ser accesibles para cualquier usuario de Internet, con independencia de los programas o del ordenador que utilice.

- Aspectos estéticos y afectivos (color, cabeceras, gráficos, etc.). Este criterio se refiere a la impresión que proporciona el documento, si es bonito, divertido, ameno, etc. ¿Hacen la lectura más fácil o más difícil? 
- ¿Se proporciona la información en muchos formatos, como FTP, Gopher, e-mail, CD-ROM, etc.?.

\section{Conclusiones}

Antes de llevar a cabo una búsqueda en Internet, es necesario definir las necesidades de información y decidir qué fuentes pueden ser las mejores para satisfacer con éxito las necesidades de información.

Todo tipo de información necesita ser evaluada según criterios de autoridad, conveniencia y otros criterios personales; no utilizar nunca información que no se pueda verificar. El establecer y aprender a utilizar criterios que filtren la información encontrada en Internet es un buen comienzo para llegar a convertirse en un consumidor crítico de la información en todas sus formas.

Debido a la naturaleza dinámica y cambiante de Internet, los recursos deberán ser re-evaluados periódicamente para determinar si aún satisfacen los criterios.

Muchas veces, las mejores fuentes de información no están disponibles de forma gratuita en la Web, ya que los productores de información cobran por esa información (bases de datos, revistas, etc.), y hay que pagar si se quieren usar estos recursos de información. Tener siempre presente que la información que conseguimos a través de Internet no es gratuita: el tiempo de conexión tiene un coste, y el tiempo que nosotros invertimos también tiene un precio, y por tanto, representa un coste, aunque sea intangible y muchas veces no se tenga en cuenta.

\section{Bibliografía}

Alexander, J.; Marsha, T. (1996). Teaching critical evaluation skills for World Wide Web resources. URL: <http://www.science.widener.edu/ withers/webeval.htm>. Fecha de consulta: 22-12-98.

American Libary Association. (1998). Evaluation criteria rating system for Web sites. URL: <http://www.ala.org/ICONN/rating.html>. Fecha de consulta: 8-1-99

Argus Associates, Inc. (1999). Clearinghouse: Ratings System. URL: <http://www.clearinghouse.net/ratings.html>. Fecha de consulta: 15-1-99.

Auer, N. (1998). Bibliography on evaluating Internet resources. URL: <http://refserver.lib.vt.edu/libinst/critthink.htm>. Fecha de consulta: 10-12-98.

Beck, S. (1997). Evaluation Criteria. The Good, The Bad \& The Ugly: or, why it's a good idea to evaluate web sources. URL: <http://lib.nmsu.edu/staff/ susabeck/evalcrit.html>. Fecha de consulta: 7-1-99.

Bopp, R. E.; Smith, L. C. (1995). Reference and Information Services: an introduction. 2nd. ed. Englewood: Colorado : Libraries Unlimited. p. 298-300. 
Brandt, D. S. (1996). Evaluating information on the Internet. URL: <http://throplus.lib.purdue.edu/ techman/evaluate.htm>. Fecha de consulta: 22-1298.

Caywood, C. (1995). Library selection criteria for WWW resources. URL: <http://www6.pilot.infi.net/ carolyn/criteria.html >. Fecha de consulta: 8-1-99.

Ciolek, T. M. (1996). Information quality : Some definitions. URL: <http://www.ciolek.com/WWWVLPages/Qltypages/QltyDefinitions.html>. Fecha de consulta: 14-199.

Ciolek, T. M. (1996). The six quests for the electronic grail : Current approaches to information quality in WWW resources. URL: <http://Www.ciolek.com/ PAPERS/QUEST/QuestMain.html>. Fecha de consulta: 7-1-99.

Collection Development Committe (1997). Checklist for evaluating Web sites. URL: <http://www.canisius.edu/canhp/canlib/webcrit.htm>. Fecha de consulta: 18-1-99.

Descy, Don E. (1998). Evaluating Internet Based Information. [Online]. URL: <http://www.lme.mankato.msus.edu/class/629/cred.htm>. Fecha de consulta: 22-1298.

Edwards, J. (1998). The good, the bad and the useless: evaluating Internet resources. URL: <http://www.ariadne.ac.uk/issue16/digital/intro.html>. Fecha de consulta: 2212-98.

Edwards , J. (1998). Tips for evaluating a World Wide Web search. URL: <http://www.uflib.ufl.edu/hss/ref/tips.html>. Fecha de consulta: 22-12-98.

Engle, M. (1996). Evaluating Web sites: criteria and tools. URL: <http://www.library.cornell.edu/okuref/research/webeval.html>. Fecha de consulta: 7-1-99.

Fenton, S. (1997). Information quality: is the truth out there?. URL: $<$ http://ils.unc.edu/ fents/310/>. Fecha de consulta: 7-1-99.

Grassian, E. (1997). Thinking critically about World Wide Web resources. URL: <http://www.library.ucla.edu/libraries/college/instruct/web/critical.htm>. Fecha de consulta: 7-1-99.

Harris, R. (1997). Evaluating Internet Research Sources. [Online]. URL: <http://www.sccu.edu/faculty/R_Harris/evalu8it.htm>. Fecha de consulta: 22-12-98.

Hoffbauer, M. (1998). How to evaluate Internet sources. Questions. URL: <http://members.aol.com/xxmindyxx/evaluate/question.html>. Fecha de consulta: 7-1-99.

Hogue, E.; Kellog, Z.; Mc Dermott, M. (1999). Checklist for evaluating web resources. URL: <http://library.usm.maine.edu/guides/webeval.html>. Fecha de consulta: 2-399.

Jacobson, T.; Cohen, L. (1996). Evaluating Internet resources. URL: <http://www.albany.edu/library/internet/evaluate.html>. Fecha de consulta: 7-1-99.

Janicke, L. (1995). Resource selection and information evaluation. URL: <http://alexia.lis.uiuc.edu/ janicke/Evaluate.html>. Fecha de consulta: 22-12-98.

Kirk, E. (1996). Evaluating information found on the Internet. URL: <http://milton.mse.jhu.edu:8001/research/education/net.html>. Fecha de consulta: 22-12-98. 
Kotlas, C. (1997). Evaluating Web Sites for educational uses: bibliography and cheklist. URL: <http://www.unc.edu/cit/guides/irg-49.html>. Fecha de consulta: 22-12-98.

Kwan, J. (1997). Criteria for evaluating information resources. URL: <http://wwwlib.usc.edu/Info/Sci/pubs/criteval.html>. Fecha de consulta: 22-12-98.

Norem, M. (1998). Evaluating Internet sites - quick guide. URL: <http:// wwwnhc.nhmccd.edu/public/lrc/research/selectsources.htm>. Fecha de consulta: 7$1-99$.

Ormondroyd , J.; Engle, M.; Cosgrave, T. (1996). How to critically analyze information sources. URL: <http://www.library.cornell.edu/okuref/research/skill26.htm>. Fecha de consulta: 7-1-99.

Patterson, S. (1997). Evaluating and citing Internet resources. URL: <http://www.udmercy.edu/htmls/Academics/library/webpage>. Fecha de consulta: 15-1-99.

Rettig, J. (1996). Beyond «cool». Analog models for reviewing digital resources. URL: <http://www.onlineinc.com/onlinemag/SeptOL/rettig9.html>. Fecha de consulta: 22-12-98.

Tichmond, B. (1996). Ten C's for evaluating Internet resources. URL: <http://www.uwec.edu/Admin/Library/10cs.html>. Fecha de consulta: 7-1-99.

Scholz, A. (1996). Evaluating World Wide Web information. URL: <http://thorplus.lib.purdue.edu/research/classes/gs175/3gs175/evaluation.html>. Fecha de consulta: 8-10-98.

Smith, A. (1996). Selection criteria for Internet information resources: a poll of members of info-quality-1. URL: <http://www.vuw.ac.nz/ agsmith/evaln/poll.htm>. Fecha de consulta: 22-12-98.

Smith , A. (1997). Criteria for evaluation of Internet information resources. URL: <http://www.vuw.ac.nz/ agsmith/evaln/>. Fecha de consulta: 22-12-98.

Solock, J. (1996). The Internet: Window to the world or hall of mirrors?. Information quality in the networked environment. URL: <http://rs.internic.net/nic-support/nicnews/nov96/enduser.html>. Fecha de consulta: 7-1-99.

Tillman, H. N. (1997). Evaluating quality on the net. URL: <http://www.tiac.net/users/hope/finqual.html>. Fecha de consulta: 22-12-98.

Tyburski, G. (1997). Publishers wanted, no experience necessary: Information quality on the Web. URL: <http://www.llrx.com/columns/quality.htm>. Fecha de consulta: 2212-98.

Wilkinson, G.; Oliver, K.; Bennet, L. (1997). Evaluating the quality of Internet information sources. URL: <http://itech1.coe.uga.edu/faculty/gwilkinson/webeval.htm>. Fecha de consulta: 4-1-99.

Scire. $5: 2$ (jul.-dic. 1999) 99-113. 